\title{
Dieter Birnbacher
}

Institut für Philosophie

Heinrich Heine Universität Düsseldorf

dieter.birnbacher@uni-duesseldorf.de

\section{WEISHEIT VS. LEBENSWEISHEIT - MIT EINEM SEITENBLICK AUF SCHOPENHAUER}

\begin{abstract}
Zusammenfassung
Weisheit wird gesucht, weil in einer zunehmend komplexer und intransparenter werdenden Welt Vertrauenswürdigkeit gesucht wird. Vertrauenswürdigkeit ist ihrerseits abhängig von der der jeweiligen Person oder Institution zugeschriebenen Unabhängigkeit. Der Beitrag argumentiert, dass sich der Begriff der Weisheit einer expliziten Definition entzieht und als Clusterbegriff mit teils kognitiven, teils psychologischen, teils moralischen Elementen gefasst werden sollte. Durch die letzteren Elemente unterscheidet sich Weisheit wesentlich von Lebensweisheit, wie sie Epikur und in seiner Nachfolge Schopenhauer verstehen. Sie sind für den „weisen Richter” charakteristischer als für den ,weisen Ratgeber”. Die Frage, wie weit Schopenhauer, einer der meistgelesenen modernen Weisheitslehrer, selbst als weise gelten kann, wird am Ende skeptisch beantwortet: Schopenhauer erfüllte viele, aber nicht alle Bedingungen, die an Weisheit zu stellen sind.
\end{abstract}

Schlüsselwörter:

Schopenhauer, die Weisheit

\section{EINLEITUNG: WEISHEIT - EIN OBJEKT DER BEGIERDE}

Weisheit ist begehrt. Weisheit ist ein gesellschaftliches Bedürfnis. Allerdings: Seitdem sich die Philosophen nicht mehr als Gurus gebärden, ist das gesellschaftliche Bedürfnis nach Weisheit - nach einem wissenden, einsichtigen, weitsichtigen und vor allem unparteiischen Urteil - zunehmend schwerer zu befriedigen. In meiner Jugend war das noch anders. Für mich und meinesgleichen waren Theodor W. Adorno und Ernst Bloch Horte der Weisheit, die man in intellektuellen Zweifelsfragen zu Rate ziehen konnte und die die Orientierung des eigenen Denkens mehr oder weniger vorgaben. Für andere waren das zur selben Zeit Karl Jaspers, Martin Heidegger oder Carl Friedrich von Weizsäcker. Sie waren das, was Schopenhauer für Nietzsche war: nicht nur Lehrer, sondern Erzieher. Hinzu kam, dass diese Denker keine Bedenken 
dagegen hatten, dass andere aus ihren Schriften und Worten als Quellen der Weisheit schöpften. Beide kannten keine falsche Bescheidenheit.

Von der heutigen Philosophie ist diese Funktion zunehmend weniger zu erhoffen. Die Philosophie ist über weite Strecken eine technische Disziplin geworden. Sie nimmt zunehmend weniger Stellung zu Lebens- und gesellschaftlichen Fragen und stellt in ihrer Methodik zunehmend höhere Ansprüche an Vorbildung und Vertrautheit mit einer für die Mehrheit esoterischen Terminologie. Antworten auf Orientierungs- und Lebensfragen werden zunehmend bei Wissenschaftlern, Psychologen, Medizinern, aber auch bei dem vielfältigen Angebot esoterischer Autoren gesucht. Aus heutiger Sicht ist es zunehmend fraglich, ob die Philosophie ihre vorrangige Rolle als die schlechthinnige Verkörperung von Weisheit - im Niederländischen heißt sie wijsbegeerte, Weisheitsstreben - wiedererlangen wird. Im öffentlichen Bereich haben seit längerem Kollektive wie Kommissionen, Räte und andere Beratungsgremien die Rolle des philosophischen Weisen übernommen.

Weisheit ist eine zugeschriebene Qualität. Ihr entspricht auf der Seite des Zuschreibenden Anerkennung, vor allem aber Vertrauen. Weisheit wird gesucht, weil Vertrauenswürdigkeit gesucht wird, insbesondere in einer zunehmend komplexer und intransparenter werdenden Welt. Vertrauenswürdigkeit ist ihrerseits abhängig von der der jeweiligen Person oder Institution zugeschriebenen Unabhängigkeit. Insofern ist es nicht weiter verwunderlich, dass Politikern und Kirchenmännern durchweg vergleichsweise weniger Vertrauen geschenkt wird als Wissenschaftlern und Richtern. Die ersteren sind - zumindest in Demokratien - abhängig vom Zuspruch ihrer Wähler und Parteien, die letzteren von ihren Dogmen und religiösen Hierarchien. Abtrünnige und Kirchenkritiker haben es leichter, als weise zu gelten, als loyale Vertreter ihrer jeweiligen Institutionen. Bei ihnen ist weniger zu vermuten, dass ihre Gebundenheit einem unabhängigen und unparteiischen Urteil in die Quere kommt. Vom Bundespräsidenten wird eher Weisheit erwartet als von den Regierungsmitgliedern. Umfragen bestätigen, dass das Bundesverfassungsgericht einerseits, die Bundesbank andererseits ein sehr viel höheres Vertrauen genießen als Rechts- oder Wirtschaftspolitiker. Die Mitglieder beider Institutionen werden nicht gewählt, sondern ernannt. Sie brauchen nicht um die Gunst des Publikums zu buhlen. Sie dienen keinem Parteiinteresse, sondern sind ausschließlich dem Allgemeinwohl verpflichtet. Ihnen wird in höherem Maße ein unabhängiges, authentisches und verlässliches Urteil zugebilligt.

\section{WAS IST WEISHEIT?}

Wir können festhalten: Wenn Weisheit die höchste philosophische Tugend ist, ist Unabhängigkeit die dafür wichtigste Teiltugend. Wer als weise gelten will, darf keine Interessenstrategie verfolgen, er darf weder im eigenen noch im 
fremden Namen Interessen Raum geben. Idealerweise sollte er nicht einmal ein Interesse daran haben, als weise zu gelten. Für die eigene Weisheit zu werben, wäre bereits zu viel des Interesses. Weise im eminenten Sinne ist der, dem andere vertrauen, ohne dass er es darauf anlegt, das Vertrauen anderer zu gewinnen.

Aber Unabhängigkeit und die daraus fließende Vertrauenswürdigkeit ist nicht schon die ganze Weisheit. Sie ist ein notwendiges, aber kein hinreichendes Element. Was der Weise darüber hinaus an Qualitäten besitzen muss, um als solcher gelten zu können, ist allerdings nicht leicht zu sagen, jedenfalls nicht leicht in eine Definition zu fassen. Das hat der Begriff der Weisheit mit anderen Perfektionsbegriffen wie Meisterschaft, Vollkommenheit oder Genialität gemeinsam.

Angesichts dieser Schwierigkeit liegt es nahe, den Begriff der der Weisheit anders als durch explizite verbale Definition zu erklären, etwa durch prototypische Exemplifikation oder durch Ostension. Als ein Paradigma von Weisheit kommt etwa die platonische Sokrates-Figur in Frage: die Figur eines zutiefst unabhängigen Denkers, dem seine Unabhängigkeit so wichtig war, dass er bereit war, dafür mit dem Tod zu bezahlen. Aber selbst dann, wenn wir uns darauf einigen könnten, Sokrates als Modell von Weisheit zu betrachten - eine ganze Reihe von Charaktermerkmalen, die er in den platonischen Dialogen manifestiert, stehen dem entgegen -, wäre damit immer noch offen, in welchen Hinsichten andere Sokrates nahekommen müssen, um ebenfalls als weise gelten zu können. Offenkundig reicht dafür der Habitus eines sokratischen Weisen - Nonkonformismus, Kritikfähigkeit, Einzelgängertum - nicht hin. Denn dieser Habitus ist von vielen nachgeahmt worden, ohne dass sie bereits deshalb als Weise gelten können. Wie immer man etwa Fritz Mauthner, den „Buddha vom Bodensee”, einschätzt: Falls er ein Weiser war, war es nicht allein wegen seines Habitus.

Noch weniger dürfte bloße Ostension hinreichen, um einen Weisen unter vielen Unweisen zu erkennen, etwa nach dem häufig zitierten, ursprünglich auf Pornografie gemünzten Wort „I know it when I see it”. Schopenhauer allerdings scheint dieser Ansicht gewesen zu sein, denn er schreibt in $\S 29$ der Parerga und Paralipomena in Bezug auf Weisheit:

Demnach ist die Anekdote durchaus glaublich, welche Squarzafichi, in seinem Leben Petrarka's, dem diesem gleichzeitigen Joseph Brivius nacherzählt, daß nämlich einst, am Hofe der Visconti, als unter vielen Herren und Edelen auch Petrarka dastand, Galezzo Visconti seinem damals noch in Knabenalter stehenden Sohne, nachmaligen ersten Herzog von Mailand, aufgab, unter den Anwesenden den weisesten herauszusuchen: der Knabe sah sie alle eine Weile an: dann aber ergriff er die Hand des Petrarka und führte ihn dem Vater zu, unter großer Bewunderung aller Anwesenden. Denn so deutlich drückt die Natur den Bevorzugten der Menschheit den Stämpel ihrer Würde auf, daß ein Kind es erkennt. ${ }^{1}$

\footnotetext{
${ }^{1}$ Arthur Schopenhauer, „Parerga und Paralipomena,” Bd. 6, in Arthur Schopenhauer, Sämtliche Werke, hg. von Arthur Hübscher. 4. Aufl. (Wiesbaden: Wissenmedia, 1988), 678.
} 
Diese behauptete Leichtigkeit, einen Weisen zu erkennen, entspricht der Erfahrung nur sehr unvollkommen. Zwar habe ich persönlich die Erfahrung gemacht, als Professor unter vielen Nicht-Professoren erkannt zu werden. Aber es wäre unvorsichtig zu sagen, alle Professoren seien eo ipso weise.

Eine dritte und nach aller Erfahrung zielführendere Weise, einen schwer zu fassenden Begriff zu explizieren, ist die Methode, ihn heuristisch zunächst als Clusterbegriff zu behandeln und einige der Merkmale aufzusuchen, die, jeweils verschieden gruppiert, Kandidaten dafür sind, hinreichende semantische Bedingungen für Weisheit zu sein. Dabei lassen sich in der Regel einige Merkmale aufweisen, die in allen solchen Clustern enthalten sind und insofern notwendige Bedingungen für die Anwendung des jeweiligen Zielbegriffs konstituieren.

Eine derartige Bedingung scheint das bereits erwähnte Merkmal der Unabhängigkeit des Urteils des Weisen von allen partikulären Interessen, eigenen oder fremden. Weisheit ist eine Sache besonderer Urteilskraft, und das, was das Urteil des Weisen primär auszeichnet, ist Unabhängigkeit. Deshalb sind Modellformen des Weisen einerseits der weise Richter, andererseits der weise Ratgeber. Eine mythologische Figur, die beide Modelle in einer Person vereinigt, ist der Nestor der homerischen Mythologie - gelegentlich auch ,der erste Weise der Literaturgeschichte" genannt. Er spielt sowohl die Rolle eines Ratgebers (z. B. für Agamemnon) als auch die eines Schlichters (z. B. im Streit zwischen Agamemnon und Achilles). Nestor verkörpert zugleich einige weitere Merkmale, die mit Weisheit assoziiert sind, etwa ein vorgerücktes Alter. Nestor wird erst im Alter zum Weisen, nachdem er lange Jahre hindurch Erfahrungen als König und Kriegsführer erworben hat. Das Vertrauen, das ihm geschenkt wird, verdankt sich in erster Linie seinem kumulierten Wissen und Können, seinem Erfahrungsschatz und seiner durch langjährige Übung erworbenen Kompetenz in Sachen Kriegskunst, Verhandlungsführung und Mediation.

In der Figur Nestors treten damit die kognitiven Momente der Weisheit in den Vordergrund. Kognitive Kompetenzen gehören wesentlich zum Weisheitsbegriff. Weisheit hat nicht nur etymologisch mit Wissen zu tun, Wer als weise gelten will, muss über Wissen verfügen. Ein unfreiwilliger Hochstapler wie der Gärtner Mr. Chance in der Filmsatire Willkommen Mr. Chance, der sich zeitlebens innerhalb des Grundstücks seines Arbeitgebers aufgehalten hat und die Welt nur aus dem Fernsehen kennt, kann nicht ernstlich als weise gelten, auch wenn er wegen der Gemeinplätze, die er kindlich-unschuldig äußert, für weise gehalten und am Ende sogar zum Präsidenten der Vereinigten Staaten vorgeschlagen wird. Weisheit erfordert in der Regel fundiertes Wissen, und zwar mehr als bloßes Bücherwissen. Weisheit erfordert auch mehr als bloßes Fachwissen und bloße Facherfahrung. Sie erfordert Weltweisheit und Welterfahrung, wörtlich verstanden, über alle Schul- oder Buchweisheit hinaus. In diesem Punkt war insbesondere Schopenhauer apodiktisch: 
Die Gelehrten sind Die, welche in den Büchern gelesen haben: die Denker, die Genies, die Welterleuchter und Förderer des Menschengeschlechts sind aber Die, welche unmittelbar im Buche der Welt gelesen haben. ${ }^{2}$

In der Regel ist deshalb Weisheit in der Tat an ein bestimmtes Lebensalter gebunden, zumindest an diejenige Lebensspanne, die notwendig ist, um auf eine substanzielle Lebenserfahrung, Welt- und Menschenkenntnis zurückgreifen zu können. Die Klausel ,in der Regel” deutet allerdings bereits darauf hin, dass es zweifelhaft ist, ob dieses Merkmal mehr denn als eine „Familienähnlichkeit” im Wittgenstein'schen Sinne gelten kann. Die Möglichkeit eines puer senex, der bereits in einem frühen Lebensalter und ohne viel von der Welt gesehen zu haben, in einem unverfälschten Sinn weise ist, scheint mir nicht kategorisch ausgeschlossen.

Unter den kognitiven Fähigkeiten ist Wissen und Erfahrung zwar das naheliegendste Merkmal des Weisen, aber doch zweifellos nicht das charakteristischste. Unter den Menschen mit Weltwissen und Welterfahrung zeichnet sich der Weise vor allem durch ein dem Weltwissen und der Welterfahrung gleichwertiges Selbstwissen aus, und zwar ein Selbstwissen, das primär wiederum nicht aus Büchern, sondern aus lebendiger Selbsterfahrung geschöpft ist. Der Weise kennt sich besser als andere - seine Stärken und Schwächen, seine Möglichkeiten und Grenzen, und vor allem die Arten von Situationen, die ihn aus der Fassung bringen und die Sachlichkeit, Ausgewogenheit und Gerechtigkeit seines Urteils gefährden. Weisheit erfordert Nüchternheit in der Selbstbeurteilung wie in der Beurteilung anderer und ist weder vereinbar mit Selbstüberschätzung noch mit Selbstunterschätzung. Der Weise weiß, wie er sich gegen die fortwährende narzisstische Versuchung, persönliche Interessen und Betroffenheiten überproportional in sein Urteil einfließen zu lassen, wappnen kann. Er weiß es zu verhindern, dass sich eigene Belange vor die Sachlichkeit, Fairness oder Zweckdienlichkeit seines Urteils schieben.

Dass Weisheit Selbstwissen erfordert, schließt ein, dass Weisheit mit jeder Form von Selbsttäuschung unvereinbar ist, möglicherweise sogar eine gewisse Immunität gegen Selbsttäuschung erfordert. Das Urteil des Weisen ist realistisch, nüchtern, illusionslos und unbestechlich - Eigenschaften, die nach psychologischer Erkenntnis nicht jedem in gleichem Maße verfügbar sind und die interessanterweise eher depressiv strukturierten Menschen als frohgemuten Sanguinikern nachgesagt werden. Wenn das so ist, erklärt das, dass Weise in der Regel keine typischen Erfolgsmenschen sind. Dem Weisen fällt es schwerer, andere mitzureißen und zu Höchstleistungen zu motivieren, als der Narzisst, der Enthusiast und der Fanatiker. Das heißt nicht, dass er nicht dennoch auf das Leben, die Karriere oder die Politik anderer beträchtlichen Einfluss nehmen kann. Aber er ist eher ein Berater der Führungsspitze als ein Volkstribun, gruppenpsychologisch gesprochen eher ein Beta als ein Alpha.

\footnotetext{
${ }^{2}$ Ibid., 522.
} 
Noch charakteristischer als Selbstkenntnis scheint für den Weisen eine Fähigkeit, die in der Regel mit Selbstkenntnis zusammenhängt, aber auch darüber hinaus geht und zum Ausgangsmerkmal der Unabhängigkeit zurückführt: die Fähigkeit zur Selbstdistanz und zur Einnahme eines überpersönlichen Standpunkts. Der Weise verfügt sowohl über eine überdurchschnittliche Fähigkeit, sich selbst aus der Perspektive eines anderen oder anderer $\mathrm{zu}$ sehen, aber zugleich auch über eine überdurchschnittliche Bereitschaft, eine solche Perspektive einzunehmen. Die überpersönliche Sicht von Adam Smiths ,idealem Beobachter”, der sein Urteil auf die Vergegenwärtigung der Perspektiven aller von einer Handlung, Strategie oder Politik Betroffenen gründet, ist ihm eher vertraut als fremd. Weil er sich in die Perspektiven vieler anderer hineinversetzen kann, versteht er die Interessen und Betroffenheiten anderer und vermag deren Sichtweisen angemessen in sein Urteil einzubeziehen. Weisheit ist insofern in gewisser Weise das Gegenteil von Borniertheit und Kleingeistigkeit.

Die Überpersönlichkeit des Urteils des Weisen zeigt sich noch in einem anderen Merkmal: seiner Entbundenheit von Trieben und Neigungen, die die Unparteilichkeit des Urteils beeinträchtigen könnten, etwa ein ausgeprägter Sexualtrieb. Der Prototyp des Weisen ist eine Figur, die von geschlechtlichen Bedürfnissen weitgehend frei ist. Entweder er ist ein Hermaphrodit - wie Buddha oder Jesus in ihren jeweiligen Standarddarstellungen - oder er wird - wie der mythische blinde Seher Teiresias - als wechselnd männlich und weiblich vorgestellt. Auch ein zeitgenössischer Weiser wie der Dalai Lama ist alles andere als eine ausgeprägt maskuline Figur.

In der Ilias verfügt Nestor noch über eine weitere, nicht rein kognitive, Fähigkeit, die ihn zum Inbegriff des Weisen prädestiniert: Beredsamkeit. Nestor ist nicht nur ein wertvoller Ratgeber, er vermag diesen Rat auch geschickt in Worte zu kleiden, nämlich so, dass sie geeignet sind, dem Beratenen im Gedächtnis zu bleiben. Beredsamkeit scheint wie Erfahrung und Alter ein typisches und charakteristisches, aber kein schlechthin notwendiges Merkmal von Weisheit. Typischerweise ist die Weisheit des Weisen keine rein privatsprachliche Angelegenheit. Sie äußert sich, und zwar in einem sozialen Kontext, wobei dieser von einer einzelnen Person bis zur Weltöffentlichkeit reichen kann. Der Prototyp weisen Sprechens ist dabei die wohlgesetzte, gemessene und verhalteneindringliche Rede. Ihren treffendsten Ausdruck hat sie für mein Dafürhalten in musikalischer Form gefunden, in dem Stück „Der Dichter spricht” in Schumanns Kinderszenen. Als Hörer kann man nicht anders als diesen Dichter sprechen zu hören, auch wenn er wortwörtlich nichts sagt. Sein Sprachrhythmus ist gemessen, tiefempfunden, väterlich, gütig, vor allem aber weise. Sein Urteil ist abschließend, ihm ist nichts Wesentliches mehr hinzuzufügen. Auf diese letzte „Kinderszene” kann keine weitere folgen. 


\section{IST WEISHEIT DASSELBE WIE "LEBENSWEISHEIT”?}

Weisheit ist mehr und anderes als Lebensweisheit, sofern man unter dieser, so wie es Schopenhauer in seinen Aphorismen zur Lebensweisheit tut, Lebensklugheit versteht, entweder im Sinne praktischer Lebensklugheit oder im Sinne theoretischer Anleitung dazu. Schopenhauers bekannteste und verbreitetste unter seinen Schriften (sie ist in nahezu alle Sprachen der Welt übersetzt worden) beginnt damit, dass er erklärt, "Lebensweisheit” im ,immanenten" Sinne verstehen zu wollen, nämlich als

[...] Kunst, das Leben möglichst angenehm und glücklich durchzuführen, die Anleitung zu welcher auch Eudämonologie genannt werden könnte: sie wäre demnach die Anweisung zu einem glücklichen Daseyn. Dieses nun wieder ließe sich allenfalls definieren als ein solches, welches, rein objektiv betrachtet, oder vielmehr (da es hier auf ein subjektives Urteil ankommt) bei kalter und reiflicher Überlegung, dem Nichtseyn entschieden vorzuziehen wäre. ${ }^{3}$

Lebensweisheit in diesem Sinn ist die Kunst der klugen Lebensführung im Sinne der Erreichung eines möglichst hohen Glücksniveaus. Sie entspricht dem, was man im Griechischen phronesis genannt hat und im Englischen prudence nennt. Ist Lebensklugheit in diesem Sinne ein konstitutiver Teil von Weisheit? Aristoteles zitiert in der Nikomachischen Ethik eine zu seiner Zeit verbreitete Auffassung, nach der Lebensklugheit nicht nur kein Teil von Weisheit ist, sondern Weisheit und Lebensweisheit sich sogar wechselseitig ausschließen:

Die Leute nennen Anaxogoras, Thales und derartige Menschen zwar weise, aber nicht klug, wenn sie sehen, dass diese das ihnen Förderliche nicht kennen; und daher sagt man auch, dass sie Dinge wissen, die außergewöhnlich, wunderbar, schwierig und göttlich sind, aber unbrauchbar, weil sie nicht die Güter für den Menschen (anthropinon kalon) suchen. ${ }^{4}$

Nach dieser Auffassung ist der Weise mit zu ,hohen" und der Menschenwelt entrückten Gegenständen beschäftigt, um als Lebensberater oder als Politiker zu taugen. Dieser Begriff von Weisheit entspricht aber nur unvollkommen dem in der Neuzeit üblichen Verständnis. Nach diesem modernen Begriff gehört die Kunst „weiser Ratschläge”, und nicht nur solcher für das Denken, sondern auch für das konkrete Leben, als fester Bestandteil zu den kognitiven Kompetenzen, die ein Weiser aufweisen muss, um als solcher gelten zu können. Als die Kunst der Befolgung und Umsetzung dieser Ratschläge verstanden, geht sie zugleich über die kognitiven Bedingungen hinaus und weist auf die nicht-kognitiven Elemente der Weisheit hin: Als weise kann nur gelten, wer nicht nur anderen den richtigen Weg zu weisen vermag, sondern der auch selbst den von ihm für richtig gehaltenen Weg geht, oder dies doch zumindest ernstlich versucht.

\footnotetext{
${ }^{3}$ Schopenhauer, „Parerga und Paralipomena,” Bd. 5, 333.

${ }^{4}$ Aristoteles, Nikomachische Ethik. Übersetzt von Ursula Wolf (Reinbek: Rowohlt Taschenbuch, 2006), 203.
} 
Dass Lebensweisheit alias Lebensklugheit nicht nur ein konstitutiver Teil von Weisheit ist, sondern auch inhaltlich mit ihr eng verwandt ist, zeigt sich in einigen Zügen, die beide miteinander gemeinsam haben.

Die erste und auffälligste Gemeinsamkeit besteht darin, dass beide Formen von Rationalität sind und in ihrem Vollzug einen gewissen Verzicht auf die Befriedigung von Augenblicksimpulsen erfordern. Die Assoziierung von Weisheit mit Impulskontrolle, Leidenschaftslosigkeit und Abgeklärtheit gilt für Weisheit ebenso wie für Lebensklugheit. Menschen mit ausgeprägter Affektdominanz haben es mit beiden schwer. Epikur, der philosophische Nestor der Lebensklugheit, hat in seine Weisheitslehre deshalb ähnliche emotionskritische Momente aufgenommen wie die Stoiker: Der Lebensweise befriedigt seine Augenblicksimpulse nur dann, wenn er sicher ist, dass sie sich nicht nachteilig auf spätere Befriedigungen oder Glückspotenziale auswirken. Gegenwärtiges Übel muss gegen späteres zeitneutral abgewogen werden ebenso wie heutiges Wohlsein gegen spätere Leiden. Leitbild der Lebensklugheit ist das „wohlverstandene Eigeninteresse” mit der Betonung auf „wohlverstanden”. Insofern entspricht das horazische „Carpe diem!” Epikurs Lehre nur unvollständig. Auch die wenig genießbare Frucht eines Tages ist nicht zu verschmähen, wenn sie zur Folge hat, dass spätere Früchte besonders köstlich sind. Der wahre Epikureer lebt weder als Genussmensch noch als Asket, sondern je nachdem, wie sich das eine oder andere auf sein Lebensglück auswirkt, nach einem rationalen Chancen-RisikoKalkül. So ist es nur konsequent, dass auch der im Allgemeinen Mäßigung empfehlende Epikur nichts dagegen hat, dass wir uns gelegentlich etwas gönnen:

Sich also zu gewöhnen an einfache und nicht aufwendige Mahlzeiten befähigt zu voller Gesundheit, macht den Menschen unbeschwert gegenüber den notwendigen Anforderungen des Lebens, stärkt unsere Verfassung, wenn wir uns in Abständen zu aufwendigen Mahlzeiten aufmachen und entläßt uns angstfrei gegenüber dem Zufall. ${ }^{5}$

Nach diesem Zitat ist Mäßigung sogar geradewegs ein Mittel zum vollendeten Lebensgenuss: Erst Enthaltsamkeit macht gelegentliche Selbstverwöhnung möglich.

Die zweite Gemeinsamkeit zwischen Weisheit und Lebensweisheit besteht darin, dass beide Formen von Rationalität nicht vollständig im Muster der Zweck-Mittel-Rationalität aufgehen. Vielmehr werden mit den Mitteln auch die Zwecke selbst einer abwägenden Prüfung unterworfen. Bei beiden geht es nicht darum, die Wahl der richtigen, besten oder bewährtesten Mittel für beliebige Ziele bzw. die Wahl der richtigen, besten oder bewährten Zwischenziele auf dem Weg zu einem gegebenen Letztziel anzuleiten, sondern darum, zur Wahl der richtigen, besten oder bewährtesten Kombination von Zielen und Mitteln

\footnotetext{
${ }^{5}$ Epikur, Briefe. Sprüche. Werkfragmente. Griechisch/Deutsch (Stuttgart: Reclam, Philipp, jun. GmbH, Verlag, 1980), 69.
} 
anzuleiten. Nicht nur Weisheit, sondern auch Lebensklugheit ist mehr als Lebenstechnik im Sinne einer rein strategischen Disziplin. Da „Glück”, „Lebenserfüllung” oder ,gutes Leben” als Zielvorgaben zu unbestimmt sind, um darauf Lebensstrategien zu gründen, bedarf Lebensweisheit einer mehr als bloß instrumentellen Rationalität, wie sie sich in der Verwendung von „wise” und „unwise” im Englischen eingebürgert hat. In diesem rein instrumentellen Sinn kann eine bestimmte Entscheidung ,wise" oder ,unwise” sein ganz unabhängig von der Weisheit oder Unweisheit der Zielbestimmung, vergleichbar den Prädikaten ,gut beraten" und ,schlecht beraten" im Deutschen. Auch dann, wenn das Ziel im höchsten Maße unvernünftig und irrational ist, kann eine darauf gerichtete Entscheidung als „wise” bezeichnet werden. Dieser rein strategische Sinn ist jedoch gerade nicht gemeint, wenn von Weisheit die Rede ist. Andernfalls würde sogar noch de Sades Philosophie du boudoir mit ihrer Empfehlung ausgeprägt ,sadistischer” Sexualpraktiken als Weisheitslehre durchgehen können.

Statt als einseitige Rationalität von Mitteln in Bezug auf gegebene Zwecke wird Lebensweisheit insofern adäquater als kluge Ausbalancierung von Mitteln und Zwecken gefasst. Die Weisheit in der Lebensweisheit besteht nicht (nur) darin, die richtigen Mittel für gegebene Zwecke zu setzen, sondern auch darin, angesichts gegebener Mittel die richtigen Zwecke zu setzen. Nicht alle Zwecke sind ja angesichts beschränkter Mittel für die Lebensgestaltung gleichermaßen geeignet. Allzu ehrgeizige Ziele bergen das Risiko von Überforderung, allzu bescheidene das Risiko von Unterforderung.

Dass Lebensklugheit auch in der Wahl der richtigen Zwecke und nicht nur in der Wahl der besten oder geeignetsten Mittel zur Zweckerreichung besteht, wird auch in den Weisheitslehren der Antike, insbesondere bei Epikur hervorgehoben. Schopenhauer ist ihm darin gefolgt. Epikur macht die Ataraxie zum Zweck in der Überzeugung, dass jede weitergehende Zwecksetzung, etwa die hedonistische des maximalen Lustgewinns, die Erreichbarkeit des Zwecks gefährden oder den Menschen überfordern würde. Zufriedenheit und Seelenruhe sind, wie man heute sagen würde, ,realistischere" Ziele als maximaler Lebensgenuss. Dabei können die Mittel, die Epikur als zur Erreichung dieses Ziels für angeraten hält, zum Teil auch heute noch als gültig angesehen werden: die Minderung des Anspruchsniveaus, die Begrenzung der Bedürfnisse auf die jederzeit und leicht erfüllbaren, das Sich-Fernhalten von Politik, die Anpassung an soziale Konventionen und die Vermeidung von übermäßig tiefen Gefühlsbindungen.

Die entscheidende Differenz zwischen Weisheit und Lebensweisheit liegt in dem zusätzlichen moralischen Gehalt der Weisheit. Weisheit hat über seine kognitiven und lebenspraktischen Aspekte hinaus einen universal-altruistischen und Gerechtigkeits-Gehalt. Dieser kommt in den häufig als „Weisheitslehren” bezeichneten Lebenslehren zu kurz. Sie werden dem Eigentümlichen der Weisheit, einen überpersönlichen, perspektivenunabhängigen und allgemeingü1tigen Standpunkt einzunehmen, nur unvollkommen gerecht. 
Dies gilt für die „Aphorismen zur Lebensweisheit” Schopenhauers ebenso wie für die seines Vorgängers Epikurs, mit der Ausnahme der Stoiker, die kurzerhand - und mit nicht weniger als paradoxen Konsequenzen - das individuelle bonum mit dem moralischen bonum gleichsetzten.

Schopenhauers wie Epikurs Lebenslehre sind gleichermaßen egozentrisch. Sie kennen Verantwortung im Wesentlichen nur als Verantwortung für die Selbstsorge, nicht als Verantwortung für andere. Mitleid und Mitleidsethik spielen in Schopenhauers Aphorismen ebenso wenig eine Rolle wie in den von Epikur überlieferten - auch wenn sich unter den „Weisungen” Epikurs Beispiele wie diese finden:

Mitfühlen wollen wir mit unseren Freunden, nicht indem wir jammern, sondern in dem wir uns um sie kümmern! ${ }^{6}$

Aber ähnlich wie Spinozas Ethik und die Tradition des Buddhismus (z. B. bei Matthieu Ricard, vgl. Ricard 2009) kennt das Weisheitsideal Epikurs Altruismus und Solidarität nur als Folgewirkung von Selbstgenügsamkeit:

Wenn der Weise im Blick auf Notlagen mit anderen verglichen wird, so versteht er eher, seinen Anteil anzubieten als anzunehmen. Einen solchen Schatz der Selbstgenügsamkeit hat er gefunden. ${ }^{7}$

Danach ist Moral für die Weisheit eine willkommener spill-over-Effekt, aber kein konstitutives Element. Zwar ist der Weise notwendig auf andere bezogen: Er lebt ihnen vor, wie man leben sollte. Aber dieses Leben kennt eine moralische Verantwortung nur insoweit, als sie sich aus Gütern ergibt, die für das gute Leben des Individuums um ihrer selbst willen wünschenswert sind.

Falls jedoch der Standpunkt, von dem aus der Weise urteilt, der Standpunkt des ,idealen Beobachters” und seine Perspektive eine Perspektive jenseits aller Perspektiven, eine ,view from nowhere” ist, erscheint das zu wenig. Die für den Weisen zentrale Dimension der Unabhängigkeit hat eine unausgefüllte Lücke. Zwar macht sich der Weise als Lebenslehrer unabhängig von den kontingenten Eigentümlichkeiten seiner Person: Er berät andere, sofern sie seinen Rat suchen, in ihrem jeweiligen Interesse und zu ihrem jeweils hochgradig individualisierten Nutzen. Aber er bleibt damit doch an die Grenzen der Individualität als solcher gebunden. Sein Urteil ist zwar nicht auf das Wohl der eigenen individuellen Person, aber doch auf das Wohl einer einzigen Person bzw. einer einzigen Personengruppe gerichtet.

Dem Merkmal der Überpersönlichkeit wird das Modell des weisen Richters insofern besser gerecht als das des weisen Ratgebers. Wenn Wissen und Rationalität die höchsten dianoetischen Tugenden des Weisen sind, ist Gerechtigkeit seine höchste ethische Tugend. Deshalb wird er auch als Berater seinen über-

\footnotetext{
${ }^{6}$ Ibid., 95.

${ }^{7}$ Ibid., 89.
} 
persönlichen Standpunkt nicht aufgeben und sich nicht rückhaltlos den Interessen des Beratenen anpassen wollen. Er wird die je partikulären Zwecke des Beratenen vielmehr daraufhin prüfen, wie weit sie mit überpartikulären Zwecken wie dem bonum der Menschheit bzw. der gesamten empfindungsbegabten Welt unter Einschluss der höheren Tiere vereinbar sind und andernfalls seine Mitwirkung verweigern. Der Weise eignet sich deshalb schlecht zum Politikberater oder zum Diplomaten, wenn diese Funktionen allein darauf zielen, nationale Interessen zu befördern. John Stuart Mill, der große Stücke auf die Weisheitslehren der Antike hielt, und einer der wenigen Philosophen, die sich in der praktischen Politik betätigten (er war Abgeordneter der Liberalen im britischen Unterhaus von 1865 bis 1868), fiel dort nicht von ungefähr als Außenseiter auf. Es sagt alles, dass Gladstone, der britische Premierminister bis 1866, Mill mit dem Epitheton ,saint of rationalism" bedachte. Mill hatte sich von vornherein nur unter der Bedingung als Kandidat aufstellen lassen, dass er sich keiner Parteidisziplin unterwerfen und lediglich in eigener Person sprechen würde.

\section{SCHOPENHAUERS PHILOSOPHIE - EINE WEISHEITSLEHRE?}

Mit seiner Philosophie hat Schopenhauer eine Weisheitslehre geschaffen, die viele der Bestimmungsstücke von Weisheit zusammenführt, wenn auch nicht alle mit derselben Vollständigkeit. Eine Besonderheit dieser Philosophie ist, dass sie vielleicht gerade deshalb als ein Modellfall von Weisheit gelten kann, weil sie im Gegensatz zu den zahlreichen Unzulänglichkeiten in puncto Weisheit entwickelt worden ist, die mit der realen Person Schopenhauer untrennbar verbunden sind. Schopenhauers Philosophie stellt gewissermaßen ein Idealselbst vor, ein Ich-Ideal, das mentale Konstrukt einer Person, von der der Autor dieses Konstrukts weiß, dass er ihm nur unvollständig entspricht. Diese Art zu philosophieren ist analog dem, wie die so genannte Persona-Theorie in der Musiktheorie den musikalischen (oder generell: den künstlerischen) Ausdruck interpretiert (vgl. Rinderle 2010, 118 ff.): Die Musik drückt das emotionale Leben einer fiktiven Idealperson aus.

Diese Tendenz zur Idealisierung gilt allerdings nur für einige der Merkmale dieser Philosophie, insbesondere für die Mitleidsethik und das im vierten Buch des Hauptwerks postulierte Ideal asketischer Lebensführung. Der Person Schopenhauer war weder eine besondere Neigung zum Mitleid noch zur Askese eigen. Deren Idealisierung war - tiefenpychologisch gesprochen - so etwas wie eine Reaktionsbildung: Mitleid und Askese werden verherrlicht, gerade weil sie in der eigenen Person defizitär sind. Auch die bramabarsierende Art, in der Schopenhauer ad nauseam über seinen Rivalen Hegel herzieht, verrät einen mit Weisheit wenig verträglichen Hang zum Perseverieren und erinnert an die Neigung pathologischer Narzissten, ihr Publikum mit Hasstiraden gegen ihre Widersacher zu ermüden. Auch der Neid und die Ruhmsucht, die aus vielen 
Passagen Schopenhauers sprechen, passen nur wenig zur Gelassenheit des Weisen. Eine tiefer liegende Unvereinbarkeit könnte auch in der zumindest beim jungen Schopenhauer unübersehbaren Neigung zu einer - mit dem transzendentalen Idealismus eng verschwisterten - Tendenz zum Solipsismus zu sehen sein. Er bringt Schopenhauer sogar noch im Alter dazu, aus Kants Auffassung von der Idealität der Zeit abzuleiten, dass mit dem Untergang der eigenen Individualität im Tod auch die Zeit - und damit auch die Welt - untergeht. Mit dem Aufhören der eigenen Lebenszeit höre auch die Zeit überhaupt auf und damit die Lebenszeit aller anderen Wesen:

[...] bin ich nicht; so ist auch keine Zeit mehr. Es ist nur ein täuschender Schein, der mir eine Zeit zeigt, die fortliefe, ohne mich, nach meinem Tode. ${ }^{8}$

Eine ganze Reihe von Momenten, die für Weisheit charakteristisch sind, sind bei Schopenhauer allerdings bereits in der Person angelegt, ohne dass sie einer besonderen Idealisierung bedürften, an erster Stelle Unabhängigkeit. Keiner hat sich mit größerer Unbeirrbarkeit Kants Maxime des Selbstdenkens zu eigen gemacht. Biografisch hatte Schopenhauers Unabhängigkeitsdrang zwar gelegentlich kauzige Züge, etwa wenn er sich in systematischem Misstrauen und übertriebenem Sicherheitsbedürfnissen äußerte. Aber ohne diesen Unabhängigkeitsdrang wäre Schopenhauer wohl kaum zu dem „Denker gegen den Strom” (Hübscher 1973) geworden, der es wagte, den roten Faden des Optimismus, der die großen metaphysischen Systeme des Westens durchzieht, als realitätsfremd in Frage zu stellen. Vorstellungen wie die, dass sich ,hinter” der Erfahrungswelt eine Welt vollkommener Ideen, ein gütiger Gott oder die „reine” Vernunft verbirgt, oder dass in der Geschichte ein Heils- oder Fortschrittsprinzip waltet, sind für Schopenhauer schlichte Wunschprojektionen. Faktisch bietet die Welt das ernüchternde Bild eines Kreislaufs des Unheils. Wenn sich daraus etwas für die Transzendenz folgern lässt, dann nichts Gutes.

Weisheit beweisen Person und Philosophie Schopenhauers vor allem in seiner Religionsphilosophie: Die Religionen können zwar als phantasievolle Einkleidungen der philosophischen Wahrheit gelten und befriedigen als „Volksmetaphysik” das ",metaphysische Bedürfnis” nach Letzterklärung und Sinnfindung, das für die Gebildeten die Metaphysik erfüllt. Außerdem übernehmen sie wichtige psychosoziale Funktionen, vor allem die, das bedrängte Individuum zu beruhigen, Trost im Leiden zu spenden, den sozialen Zusammenhalt zu fördern und zusätzliche Motivation für die Moral zu liefern. Aber zugleich kleiden sie ihren Wahrheitskern in das "Gewand der Lüge", indem sie die Menschen glauben machen, die religiösen Bilder und Geschichten, die diese Wahrheit transportieren, seien wortwörtlich wahr.

\footnotetext{
${ }^{8}$ Schopenhauer, „Parerga und Paralipomena,” Bd. 5, 91.

${ }^{9}$ Schopenhauer, ,Parerga und Paralipomena,” Bd. 6, 353.
} 
Was mich allerdings zögern lässt, Schopenhauers Philosophie als ganzer Weisheit zuzubilligen, sind einerseits die große intellektuelle Abhängigkeit, in die sich Schopenhauer trotz aller Kritik in Bezug auf die Philosophie Kants begibt, andererseits das offensichtliche Unvermögen seiner Philosophie, die sich im so genannten „Hirnparadox” zuspitzende Spannung zwischen Idealismus und Realismus, Reduktion der Welt auf Vorstellungen einerseits, Physiologisierung der Verstandesfunktionen andererseits aufzulösen. Schopenhauers Philosophie findet, um einen mechanischen Vergleich $\mathrm{zu}$ bemühen, nirgendwo eine Gleichgewichtslage. Sie ist so zerrissen wie die Welt, die sie beschreibt. Dass sie möglicherweise der adäquate Ausdruck dieser Zerrissenheit ist, spricht für sie, zumindest in einem ästhetischen Sinn. Zur vollkommenen Weisheit fehlt etwas: innere Stimmigkeit, In-sich-Ruhen, Auflösung der Spannungen in einer gelassenen Synthesis. Weisheit ist nicht ohne Gelassenheit denkbar - nicht nur als Ideal, sondern als Wirklichkeit.

\section{BIBLIOGRAPHIE}

Aristoteles. Nikomachische Ethik. Übersetzt von Ursula Wolf. Reinbek: Rowohlt Taschenbuch, 2006.

Birnbacher, Dieter. „Utilitaristische Theorie und Praxis. John Stuart Mill als Politiker.” In: Hans G. Nutzinger/Herwig Unnerstall/Gottlind Ulshöfer (Hg.), Ökonomie Nach-Denken. Zur Aktualität von John Stuart Mill. Marburg: Metropolis-Verlag, 2014, 43-59.

Epikur. Briefe. Sprüche. Werkfragmente. Griechisch/Deutsch. Stuttgart: Reclam, Philipp, jun. GmbH, Verlag, 1980.

Hübscher, Arthur. Denker gegen den Strom. Schopenhauer. Gestern - heute - morgen, Bonn: Bouvier, 1973.

Ricard, Matthieu. Glück. München: Knaur MensSana TB, 2003.

Rinderle, Peter. Die Expressivität von Musik. Münster: Mentis, 2010.

Schopenhauer, Arthur. Sämtliche Werke, hrsg. von Arthur Hübscher. 4. Aufl. Wiesbaden: Wissenmedia, 1988.

\section{WISDOM VS. LEBENSWEISHEIT - WITH A SIDE GLANCE AT SCHOPENHAUER}

An increasingly complex and intransparent world makes for an increasing demand for trustworthy orientation. Thence the increasing demand for wisdom. What is wisdom? Wisdom primarily depends on the independence ascribed to a person or institution, but that does not imply that the notion of wisdom is amenable to an explicit definition. It is proposed to construct it as a cluster concept with cognitive, psychological and moral elements. The difference then becomes apparent between wisdom and lebensweisheit. The latter corresponds to prudence as understood by Epicurus and, in the same tradition, Schopenhauer, and lacks the moral elements essential to wisdom. In conclusion, the question is asked how far Schopenhauer, one of the most popular teachers of lebensweisheit in modern times, can be qualified as wise. This question is given a skeptical answer. Schopenhauer meets many, but not all conditions necessary for wisdom.

\section{Keywords:}

Schopenhauer, wisdom 